\title{
Characteristics affecting fibrinolytic activity and plasma fibrinogen concentrations
}

\author{
T W MEADE, R CHAKRABARTI, A P HAINES, W R S NORTH, YVONNE STIRLING
}

British Medical fournal, 1979, 1, 153-156

\section{Summary and conclusions}

As part of a study to determine the extent to which the haemostatic system is implicated in the onset of clinically manifest ischaemic heart disease, characteristics influencing fibrinolytic activity (FA) and plasma fibrinogen concentrations were examined in 1601 men aged 18-64 and 707 women aged 18-59 in several occupational groups in North-west London. In men FA noticeably decreased till the age of about 58, when there was a small rise. In women a small increase in FA between 18 and about 40 was followed by a slightly larger fall between 40 and 59 . There was a pronounced negative association of FA with obesity. FA was significantly less in smokers than non-smokers, though the effect was not large. FA increased with alcohol consumption. FA in men appeared to be greatest in the lower social classes, and men on night shift had poorer FA than those on day work. FA was greater in women using oral contraceptives than in those not using these preparations. In both sexes $F A$ increased with exercise, but there were no associations between any of the characteristics studied and the increase.

Plasma fibrinogen concentrations increase with age and obesity, are higher in smokers than non-smokers, and fall with alcohol consumption. In women the concentrations are higher in those using oral contraceptives. The general epidemiology of FA and plasma fibrinogen concentrations suggests that they may well be implicated in the pathogenesis of ischaemic heart disease.

\section{Introduction}

Clinical observations suggest that poor fibrinolytic activity $(\mathrm{FA})^{12}$ and high plasma fibrinogen concentrations ${ }^{3}{ }^{4}$ may be implicated in the onset and course of thromboembolic disease. Epidemiological evidence, however, is scarce. Prospective data on associations of FA and fibrinogen concentrations with ischaemic heart disease (IHD) will eventually be available from the Northwick Park Heart Study (NPHS). ${ }^{5}$ Meanwhile crosssectional data at recruitment to the study provide an opportunity for seeing whether the general epidemiology of FA and fibrinogen concentrations is consistent with their having a role in the pathogenesis of IHD. If these variables are influenced by personal and environmental factors-such as age, smoking, and obesity-already known to be associated with an increased risk of the disease the case for such a role would be strengthened. In turn there would be added reason to consider the contribution to IHD of thrombogenic mechanisms as well as those concerned

\footnotetext{
MRC-DHSS Epidemiology and Medical Care Unit, Northwick Park Hospital, Harrow, Middlesex HA1 3UJ

$T$ W MEADE, BM, MRCP, director

R CHAKRABARTI, MB, MRCPATH, member of scientific staff

A P HAINES, MB, MRCP, member of scientific staff

W R S NORTH, MA, MSC, member of scientific staff

YVONNE STIRLING, FIMLS, head technician
}

with lipids and blood pressure. We therefore describe several personal and environmental characteristics influencing FA and plasma fibrinogen concentrations in participants in the study.

\section{Study population and methods}

Full details of the population being studied and methods used have been reported. ${ }^{5}$ The population consists of members of several occupational groups in North-west London. This report deals with only white participants-namely, 1601 men aged 18-64 and 707 women (88 users of oral contraceptives) aged 18-59. FA is expressed as $100 /$ dilute clot lysis time $e^{6}$ in hours. Thrombin-"clottable" fibrinogen is measured gravimetrically ${ }^{7}$; it is $\log$-normally distributed, ${ }^{5}$ and all mean values given here are geometric. Blood for measurement of FA is taken before and after a spell of exercise consisting in stepping on and off a chair 20 times (except in those with clinical or electrocardiographic manifestations of IHD, who are not exercised, and from whom only the pre-exercise sample is available). Pulse rates are recorded before and after exercise. Fibrinogen concentrations are determined in the pre-exercise sample. All samples are taken between 0700 and 1100. The personal and environmental characteristics considered here are age, sex, smoking habit, alcohol consumption, social class, obesity, current use or otherwise of oral contraceptives in premenopausal women, and, in men, working shift (day or night).

Because many of the characteristics studied are associated with one another-for example, smoking and social class-step-wise multiple regression has been used to indicate the independent contribution of each characteristic to FA and fibrinogen concentration; these analyses are based on the number of cigarettes (or pipe or cigar equivalents) smoked and grams of alcohol consumed daily. One cigar or $1 \mathrm{~g}$ pipe tobacco is taken as equivalent to one cigarette. Alcohol consumption is elicited at interview in terms of weekly consumption but expressed as daily consumption. The figure and tables I-IV show the results of the regression analysis. In tables I and IV smokers are those smoking at least one cigarette, pipe, or cigar a day. Ex-smokers are included with non-smokers. In table I drinkers are those who recorded a regular weekly intake of alcohol, of whatever amount. Teetotallers and those whose stated intake is at less than weekly intervals are defined as non-drinkers. About $80 \%$ of weekly alcohol consumption (by weight) among male drinkers was in the form of beer. Obesity is assessed from the sum of the skinfold thicknesses at triceps, subscapular, suprailiac, and forearm sites.

Numbers of participants in different analyses vary slightly because of missing data.
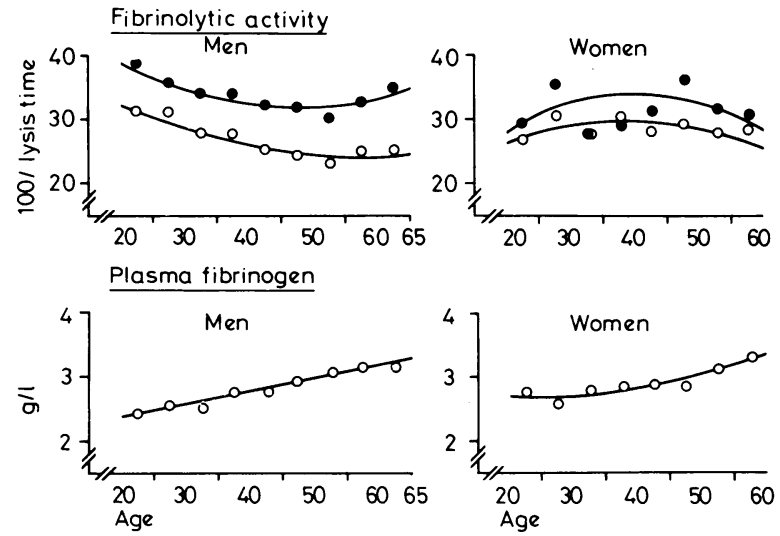

Mean fibrinolytic activities and plasma fibrinogen concentrations before $(O)$ and after $(O)$ exercise in men and women at various ages. (Minimum numbers in any five-year age group: men 87, women 42.) 


\section{Results}

The following simplified regression equations indicate the independent contributions of the various characteristics to FA and fibrinogen concentration. Figures in square brackets are $t$ values associated with the regression coefficients.

Men:

FA $\quad($ pre-exercise $)=28 \cdot 4+0.006 \quad[6 \cdot 19] \times(\text { age }-58)^{2}-0 \cdot 1[3 \cdot 28] \times$ cigarettes $+0.07 \quad[4.31] \times$ alcohol $-0.2 \quad[10.72] \times$ skinfolds $+1.0 \quad[3.67] \times$ social class $-4[3.93] \times$ shift \pm RSD, 14.0

Fibrinogen $=1.72+0.02 \quad[16.64] \times$ age $+0.01 \quad[6.64] \times$ cigarettes -0.002 $[2 \cdot 82] \times$ alcohol $+0.005[5 \cdot 17] \times$ skinfolds \pm RSD, 0.62

Women:

FA $\quad($ pre-exercise $)=39 \cdot 2-0.01 \quad[2.98] \times(\text { age }-39)^{2}-0.2 \quad[3.34] \times$ cigarettes $-0.16 \quad[6.13] \times$ skinfolds $+6 \quad[3.58] \times$ oral contraceptive use $\pm \mathrm{RSD}, 14 \cdot 1$

Fibrinogen $=2.33+0.0005 \quad[8.46] \times(\text { age }-23)^{2}+0.006 \quad[2.24] \times$ cigarettes $-0.015[3.81] \times$ alcohol $+0.006 \quad[5.68] \times$ skinfolds $+0.24 \quad[3.23]$ $x$ oral contraceptive use $\pm R S D, 0.59$

where age $=$ age in years; cigarettes $=$ number of cigarettes (or equivalents)/day; alcohol $=\mathbf{g} /$ day, stated intake; skinfolds $=$ sum of skinfold thicknesses at four sites (mm); social class $=1-6$ (for I, II, III, non-manual, III manual, IV, V); oral contraceptive use $=0$ (no), 1 (yes); shift $=0$ (day men), 1 (night men); and $\mathrm{RSD}=$ residual standard deviation.

Where $t>1.94, \mathrm{P}<0.05 ; t>2.57, \mathrm{P}<0.01 ; t>3.34, \mathrm{P}<0.001$.

About $15 \%$ of the total variance in FA and $20 \%$ of that in fibrinogen concentrations may be explained in terms of the characteristics studied; the RSDs are large. The data were extensively analysed for effects of interactions between the various characteristics, but such interactions as there were do not materially alter the summary

TABLE I-Mean fibrinolytic activities and plasma fibrinogen concentrations in male smokers (mean $15 \cdot 7 \pm S D 10 \cdot 1$ cigarettes or equivalents daily) and nonsmokers and drinkers (mean $22 \cdot 8 \pm 22.9 \mathrm{~g}$ alcohol daily) and non-drinkers. (Numbers of men in parentheses)

\begin{tabular}{|c|c|c|c|}
\hline & Smokers & Non-smokers & Total \\
\hline \multicolumn{4}{|c|}{ Fibrinolytic activity (100/lysis time) } \\
\hline $\begin{array}{l}\text { Drinkers } \\
\text { Non-drinkers }\end{array}$ & $\begin{array}{l}25 \cdot 8(492) \\
24.0(314)\end{array}$ & $\begin{array}{l}27 \cdot 7(458) \\
26 \cdot 7(337)\end{array}$ & $\begin{array}{l}26.8(950) \\
25 \cdot 4(651)\end{array}$ \\
\hline Total & $25 \cdot 1(806)$ & $27 \cdot 3(795)$ & \\
\hline \multicolumn{4}{|c|}{ Plasma fibrinogen concentration $(g / l)$} \\
\hline $\begin{array}{l}\text { Drinkers } \\
\text { Non-drinkers }\end{array}$ & $\begin{array}{l}2.86(489) \\
3.03(308)\end{array}$ & $\begin{array}{l}2 \cdot 63(456) \\
2 \cdot 77(339)\end{array}$ & $\begin{array}{l}2 \cdot 75(945) \\
2.89(647)\end{array}$ \\
\hline Total & $2.92(797)$ & $2.69(795)$ & \\
\hline
\end{tabular}

TABLE II-Mean fibrinolytic activities and plasma fibrinogen concentrations in men of different ages and with different skinfold thicknesses (mean skinfold thickness $42 \cdot 0 \pm S D 17.0 \mathrm{~mm}$ ). (Numbers of men in parentheses)

\begin{tabular}{|c|c|c|c|c|}
\hline \multirow[b]{2}{*}{ Age (years) } & \multicolumn{3}{|c|}{ Skinfold thickness $(\mathrm{mm})$} & \multirow[b]{2}{*}{ Total } \\
\hline & $<35$ & $35-50$ & $>50$ & \\
\hline \multicolumn{5}{|c|}{ Fibrinolytic activity (100/lysis time) } \\
\hline $\begin{array}{l}18-34 \\
35-49 \\
50-64\end{array}$ & $\begin{array}{l}35.5(206) \\
30.9(169) \\
29 \cdot 7(178)\end{array}$ & $\begin{array}{l}29 \cdot 5(107) \\
24 \cdot 5(225) \\
22 \cdot 7(263)\end{array}$ & $\begin{array}{l}23.9(83) \\
20 \cdot 3(160) \\
19 \cdot 8(182)\end{array}$ & $\begin{array}{l}31 \cdot 4(396) \\
25 \cdot 2(554) \\
23.9(623)\end{array}$ \\
\hline Total & $32 \cdot 2(553)$ & $24 \cdot 6(595)$ & $20 \cdot 8(425)$ & $26 \cdot 3(1573)$ \\
\hline \multicolumn{5}{|c|}{ Plasma fibrinogen concentration $(\mathrm{g} / \mathrm{l})$} \\
\hline $\begin{array}{l}18-34 \\
35-49 \\
50-64\end{array}$ & $\begin{array}{l}2 \cdot 30(200) \\
2 \cdot 70(168) \\
3.05(172)\end{array}$ & $\begin{array}{l}2.41(106) \\
2.80(225) \\
3.03(268)\end{array}$ & $\begin{array}{l}2 \cdot 65(80) \\
2.91(157) \\
3 \cdot 14(187)\end{array}$ & $\begin{array}{l}2 \cdot 40(386) \\
2 \cdot 80(550) \\
3 \cdot 10(627)\end{array}$ \\
\hline Total & $2 \cdot 64(540)$ & $2 \cdot 82(599)$ & $2.95(424)$ & $2 \cdot 80(1563)$ \\
\hline
\end{tabular}

TABLE IV-Mean fibrinolytic activities (100/lysis time) in premenopausa women by smoking 'habit and oral contraceptive use. (Numbers of women in parentheses)

\begin{tabular}{clcc}
\hline & Smokers & Non-smokers & Total \\
\hline On oral contraceptives & $30 \cdot 3(33)$ & $37 \cdot 9(55)$ & $35 \cdot 1(88)$ \\
Not on oral contraceptives & $26 \cdot 3(111)$ & $30 \cdot 5(164)$ & $28 \cdot 8(275)$ \\
\hline Total & $27 \cdot 2(144)$ & $32 \cdot 3(219)$ &
\end{tabular}

shown in the regression equations. The inclusion of data on cholesterol and blood pressure in further multiple regression analyses added little $(1 \%$ or $2 \%$ ) to the variance in $\mathrm{FA}$ or fibrinogen concentration that could be explained.

There were no associations between the increase in FA on exercise and any of the characteristics studied, even after standardising response to exercise, as far as possible, by taking change in pulse rate into account.

The figure shows the influence of age on FA and fibrinogen after removing the effects of smoking, alcohol consumption, social class, obesity, oral contraceptive usage, and shift. For FA the regressions in both sexes are significantly curved. In men FA declines noticeably with age until about 58, after which there is, if anything, an increase. In women a small increase until the age of about 39 is followed by a decline; overall FA falls slightly with age. Post-exercise levels are about $25 \%$ higher than pre-exercise levels in men at all ages; they are also higher in women, though the pattern is less consistent. Plasma fibrinogen concentrations increase with age in both sexes; in men the line of best fit is linear, while in women it is curvilinear. (Distributions and tabulations of numerical values of FA and fibrinogen concentrations have been reported. ${ }^{5}$ )

Table I shows the influence of smoking and alcohol consumption in men; FA is lower and the fibrinogen concentration higher in smokers than non-smokers. Alcohol consumption appears to increase $\mathrm{FA}$ and lower the fibrinogen concentration. There is a difference in the fibrinogen concentration of about $0.4 \mathrm{~g} / 1$ between the high value of those who smoke but do not drink and the lower one of those who do not smoke but drink; values for non-smokers and non-drinkers, and smokers and drinkers are intermediate. Findings in women were similar,* though the effect of alcohol on FA was not significant at a conventional $5 \%$ level.

Table II shows strong associations of FA and fibrinogen concentrations with skinfold thickness in men. In the case of FA the pattern of a considerably poorer level in the more obese is entirely consistent. Only in those aged $50-64$ is there a minor exception to a consistent rise in fibrinogen concentration with increasing obesity. Again the findings in women were similar.

Table III gives the findings in men according to social class. FA is greater in the lower than upper social classes. There was no social class effect in women. Table IV shows the effects of oral contraceptive use and smoking habit on FA. The highest level is seen in those on oral contraceptives who do not smoke, and the lowest in those not on oral contraceptives who do smoke.

\section{Discussion}

There is no general agreement about the most appropriate method for assessing FA because of the difficulty of measuring activator and inhibitor levels directly. All methods use in-vitro techniques assumed to reflect the in-vivo state. One advantage of the dilute blood clot lysis time, especially in large-scale studies, is its practical simplicity. A second is that it uses whole

*Details of findings in women corresponding to those shown for men in tables I-III are available from the authors on request.

TABLE III-Mean fibrinolytic activities and plasma fibrinogen concentrations in men according to social class. (Numbers of men in parentheses)

\begin{tabular}{|c|c|c|c|c|c|c|}
\hline & I & II & $\stackrel{\text { III }}{\text { (Non-manual) }}$ & $\begin{array}{c}\text { III } \\
\text { (Manual) }\end{array}$ & IV & V \\
\hline $\begin{array}{l}\text { Mean age (years) } \\
\text { Fibrinolytic activity (100/lysis time) } \\
\text { Plasma fibrinogen }(\mathrm{g} / \mathrm{l})\end{array}$ & $\begin{array}{l}42 \cdot 7(180) \\
23 \cdot 7 \\
2 \cdot 73\end{array}$ & $\begin{array}{l}44 \cdot 1(340) \\
25 \cdot 4 \\
2 \cdot 79\end{array}$ & $\begin{array}{l}46 \cdot 0(193) \\
24 \cdot 5 \\
2 \cdot 86\end{array}$ & $\begin{array}{l}44 \cdot 5(542) \\
27 \cdot 4 \\
2 \cdot 79\end{array}$ & $\begin{array}{l}47 \cdot 8(304) \\
27 \cdot 5 \\
2 \cdot 86\end{array}$ & $\begin{array}{l}54 \cdot 5(35) \\
28 \cdot 1 \\
2 \cdot 88\end{array}$ \\
\hline
\end{tabular}


blood, and in this respect is more physiological, or less unphysiological, than other methods. Although FA measured by the dilute blood clot lysis time mainly reflects activators, inhibitors are also present. Differences between groups-for example, between older and younger men-may be due to a decrease in activators or increase in inhibitors or both; whole blood methods cannot distinguish with certainty between these possibilities (although whole blood and euglobulin lysis times are fairly closely correlated, ${ }^{9}$ euglobulin lysis times being virtually independent of inhibitors). If it is the in-vivo balance between activation and inhibition that determines the role of FA in thromboembolic disease, however, whole blood methods may actually be more relevant than those using different fractions. The laboratory measurement of FA by the dilute clot lysis method is fairly precise, ${ }^{5}$ but FA assessed in this way is subject to considerable within-person variation ${ }^{5}$; this may, however, also be true of other methods and of the fibrinolytic system as a whole.

During the later part of the recruitment period there was a fall in mean levels of FA and a small rise in mean concentrations of fibrinogen. We are not certain whether this was due to technical factors or to real biological differences between the participating groups. The pattern of our results is not affected by these considerations, however, since it is based on comparisons within the groups and not between them.

Previous studies using the dilute blood or plasma clot lysis time ${ }^{10-14}$ or other methods ${ }^{15-18}$ have disclosed either no effect of age on FA or that FA increases with age and no difference between men and women. Those studies, however, were based on between 50 and 200 participants, and because of the variability in FA they may have been too small ${ }^{8}$ to detect an age effect. Our results indicate firstly an appreciable decrease in FA in men and a clear difference between the sexes in the relation between age and FA. Secondly, there is a strong inverse association between obesity and FA; our findings confirm the overall results of other studies ${ }^{19-25}$ on fewer subjects and show the effect of obesity across a wide range of ages in both sexes. Thirdly, smoking reduces FA to some extent in both sexes. Other studies ${ }^{26}{ }_{27}$ have given inconclusive results, probably because of the relatively small numbers of participants. Fourthly, we have shown elsewhere ${ }^{28}$ that FA is lower in white than black men, though this is not true of women. Our data on the effects of age, smoking, obesity, and oral contraceptives are also consistent with fibrinogen having a role in thromboembolic disease. Although the fibrinogen concentration often appears to be increased when FA is decreased, and vice versa, there are exceptions; for example, oral contraceptives appear to raise both.

The effects of alcohol suggested by our data on both FA and fibrinogen would be expected to be beneficial so far as IHD is concerned and may, in addition to other effects, ${ }^{29}$ help to explain the apparently lower occurence of coronary artery disease ${ }^{30}$ and $\mathrm{IHD}^{31}$ associated with alcohol intake. Our findings deal with the effects of habitual consumption of mostly moderate amounts; acute effects on FA probably result in a temporary decrease in activity. ${ }^{32}$ Possibly constituents of alcoholic drinks other than alcohol itself are responsible for the effects on FA. ${ }^{32}$

It is not yet clear whether the $15 \%$ lower level of FA in night-shift men ${ }^{28}$ than day-shift men is due to diurnal or other influences.

A statistical reason for our finding that the characteristics studied apparently have no effect on the response of FA to exercise could be that the large variability in FA is incorporated twice over in calculating the difference between pre-exercise and post-exercise levels. On the other hand, the difference itself is large, especially in men, and with the substantial numbers of participants obvious effects would probably have emerged despite the variability. It is therefore not at all certain that the increase in FA after a stimulus such as ..vercise or venous occlusion ${ }^{33}$ is of any practical significance, and our results suggest that the measurement of unstimulated FA is more useful than that of "fibrinolytic potential."
We suggested elsewhere ${ }^{34}$ that the increase in FA apparently brought about by oral contraceptives may be part of a mechanism to compensate for the increase in clotting factors etc also caused by these preparations. It appeared that the increase in FA in those using oral contraceptives may be largely abolished by smoking, an observation that fits in well with the epidemiology of myocardial infarction in younger women. ${ }^{35}$ There were, however, insufficient women in our previous report to be sure whether there was an effect due to smoking as well as to oral contraceptives. The larger numbers now reported indicate a probable smoking effect on FA in women (as well as in men); the multiple regression analysis suggests that smoking and oral contraceptives act independently of one another, but in opposite directions.

Another situation where, contrary to expectations, increased rather than decreased FA is found is low social class in men; it now seems clear $^{36}$ that mortality from IHD is higher in the lower social classes. (The social class effect on FA remains $(\mathrm{P}<0.001)$ even after taking the progressive fall in mean $\mathrm{FA}$, referred to above, into account.) The explanation of the class effect is not clear; differences in levels of physical activity of occupation do not account for it. The apparently paradoxical effects of oral contraceptives and low social class on FA suggest that those whose FA does not increase under these or related stimuli may be at particular risk. While still largely speculative, the general principle seems worth further study. These considerations do not alter the basic hypothesis that impaired FA predisposes to thromboembolism, but the response of FA to different stimuli and the interpretation of these responses are likely to be more complex than has been generally thought.

In conclusion it seems very possible that the adverse effects on IHD risk of advancing age, obesity, smoking, and oral contraceptives and the potentially beneficial effect of alcohol are at least partly mediated through FA and plasma fibrinogen concentrations. How far these pathways are separate from those concerned with lipid metabolism and blood pressure can only be further assessed when prospective data are available; but correlations in NPHS data between FA or fibrinogen and lipids or blood pressure are not high, ${ }^{37}$ suggesting that poor FA and high plasma fibrinogen concentrations may well have independent effects.

We are grateful to those from $\mathrm{H} \mathrm{J}$ Heinz Co Ltd, the London Boroughs of Harrow and Brent, and the North-west London Telephone Area of the Post Office who have taken part in this study. We also thank colleagues in the unit for their help.

\section{References}

${ }^{1}$ Fearnley, G R, and Chakrabarti, R, Fournal of Clinical Pathology, 1964, $17,328$.

2 Johnson, A J, and McCarty, W R, fournal of Clinical Investigation, 1959, 38, 1627.

${ }^{3}$ Dormandy, J A, et al, British Medical fournal, 1973, 4, 576.

${ }^{4}$ Dormandy, J A, et al, British Medical fournal, 1974, 4, 259.

5 Meade, T W, and North, W R S, British Medical Bulletin, 1977, 33, 283.

- Fearnley, G R, and Chakrabarti, R, Lancet, 1962, 2, 128.

7 Fearnley, G R, and Chakrabarti, R, Lancet, 1966, 2, 757

${ }^{8}$ Meade, T W, Chakrabarti, R, and North, W R S, British Medical fournal, $1977,1,837$.

9 Flute, P T, British Medical Bulletin, 1964, 20, 195.

${ }_{10}$ Hume, R, fournal of Clinical Pathology, 1961, 14, 167

11 Swan, H T, British fournal of Haematology, 1963, 9, 311

12 Fearnley, G R, Chakrabarti, R, and Avis, P R D, British Medical fournal, 1963, 1, 921 .

${ }^{13}$ Mann, R D, fournal of Clinical Pathology, 1967, 20, 223.

${ }^{14}$ Chakrabarti, R, et al, Lancet, 1968, 1, 987.

15 Sawyer, W D, et al, fournal of Clinical Investigation, 1960, 39, 426.

$16 \mathrm{Rimnn}$. A, and Segal, S, Thrombosis et Diathesis Haemorrhagica, 1964, $11,119$.

17 Brakman, P, Albrechtsen, O K, and Astrup, T, British fournal of Haematology, 1966, 12, 74.

18 Hamilton, P J, et al, fournal of Clinical Pathology, 1974, 27, 326. 
19 Shaper, A G, et al, fournal of Atherosclerosis Research, 1966, 6, 313.

${ }^{20}$ Grace, C S, Australian Annals of Medicine, 1969, 18, 32.

21 Grace, C S, and Goldrick, R B, Fournal of Atherosclerosis Research, 1968, 8, 705 .

${ }^{22}$ Grace, C S, Clinical Science, 1968, 34, 497.

${ }^{23}$ Bennett, N B, et al, fournal of Clinical Pathology, 1966, 19, 241.

24 Warlow, C P, et al, fournal of Clinical Pathology, 1972, 25, 484.

${ }^{25}$ Korsan-Bengsten, K, et al, Thrombosis Research, 1972, 1, 389.

${ }^{26}$ Billimoria, J D, et al, Atherosclerosis, 1975, 21, 61.

27 Janzon, L, and Nillson, I M, Circulation, 1975, 51, 1120.

28 Meade, T W, et al, British Heart fournal, 1978, 40, 789.

29 Castelli, W P, et al, Lancet, 1977, 2, 153.

30 Barboriak, J J, et al, British Heart fournal, 1977, 39, 289.
31 Yano, K, Rhoads, G G, and Kagan, A, New England fournal of Medicine, 1977, 297, 405.

${ }^{32}$ Fearnley, G R, et al, Lancet, 1960, 1, 184

33 Shaper, A G, et al, British Medical fournal, 1975, 3, 571.

${ }^{34}$ Meade, T W, et al, British fournal of Haematology, 1976, 34, 351.

${ }^{35}$ Mann, J I, et al, British fournal of Preventive Medicine, 1976, 30, 94.

${ }^{36}$ Office of Population Censuses and Surveys, Occupational Mortality 1970 1972. London, HMSO, 1978.

${ }^{37}$ Meade, T W, Chakrabarti, R, and North, W R S, in Atherosclerosis, Metabolic, Morphologic and Clinical Aspects, ed G W Manning and M D Haust. New York, Plenum, 1977.

\title{
Comparative study of automatic blood-gas analysers and their use in analysing arterial and capillary samples
}

\author{
P RUBIN, S BRADBURY, K PROWSE
}

British Medical fournal, 1979, 1, 156-158

\section{Summary and conclusions}

Three automatic blood-gas analysers were compared for ease of use; calibration; reproducibility and accuracy of results; maintenance; fault-finding; and use of expert technician time. Results obtained from arterial and capillary blood were compared with duplicate values obtained with a semi-automatic analyser controlled and calibrated with tonometered blood. No analyser was fully automatic, and all three needed maintenance by expert technicians. Difficulties were encountered when inexperienced operators used the machines. One automatic blood-gas analyser gave aberrant values for oxygen pressure $\left(\mathrm{Po}_{2}\right)$ due to electrode dysfunction that was not indicated by the fault-finding system. A second analyser gave significantly lower values for blood pH than the standard machine. A comparison of $\mathrm{pH}$, carbon dioxide pressure $\left(\mathrm{PCO}_{2}\right)$, and $\mathrm{Po}_{2}$ measured in 40 simultaneous paired samples of arterial and arterialised capillary blood showed no significant difference for $\mathbf{p H}$ or $\mathrm{PCO}_{2}$, but the $\mathrm{Po}_{2}$ values were significantly lower in the capillary samples over the range studied.

We conclude that all machines perform satisfactorily in terms of blood-gas analysis, but none may be regarded as fully automatic. Some degree of technical supervision is essential, as is proper training for all potential users.

\section{Introduction}

The importance of blood-gas measurements in intensive-care medicine and paediatrics has led to the introduction of blood-gas analysers with automatic facilities. The advantage of such

North Staffordshire Hospital Centre, City General Hospital, Stoke on Trent ST4 6QG

P RUBIN, BM, MRCP, medical registrar (present appointment: research fellow, division of clinical pharmacology, Stanford University, California USA)

S BRADBURY, chief technician, department of respiratory physiology K PROWSE, MD, MRCP, consultant physician machines is that they may be sited outside the routine laboratory, close to an acute unit where frequent measurements are required, and their automatic facilities enable measurements to be made by non-laboratory staff such as doctors and senior nurses.

We report a study of three currently available automatic blood-gas analysers and compare results obtained from arterial and arterialised capillary samples.

\section{Methods}

We studied one model of each of three automatic blood-gas analysers-namely, the Corning 175 (Corning Medical); the IL 613 (Instrumentation Laboratory (UK) Limited); and the Radiometer ABL2 (Radiometer, Copenhagen). The machines were studied consecutively. We chose these three models because they were the three automatic blood-gas analysers available in this country. The machines tested were demonstration models four to six months old, which were supplied and set up in the laboratory by the manufacturers. The machines were studied for one week each, during which they were used by technicians experienced in blood-gas analysis, who tested them for stability, ease of calibration and maintenance, reproducibility of results, fault-finding, and general usage. During a subsequent two-day period they were tested by medical and nursing staff with no experience of blood-gas analysis.

Duplicate measurements of $\mathrm{pH}$, carbon dioxide pressure $\left(\mathrm{PCO}_{2}\right)$ and oxygen pressure $\left(\mathrm{PO}_{2}\right)$ were made in arterial blood and arterialised capillary blood samples taken from patients in the respiratory unit. Arterial samples were taken by percutaneous brachial artery puncture, $2 \mathrm{ml}$ of arterial blood being drawn into a heparinised plastic syringe. The samples were immediately placed in ice and the measurements made within five minutes. Arterialised capillary samples were taken by the method of Godfrey $e t a l^{1}$ and were also analysed within five minutes. The order of analysis of arterial and capillary samples was randomised. The results obtained with the three machines were compared with duplicate values obtained from the same samples with a semi-automatic machine (Corning 165), which was used by an experienced technician. The Corning 165 was calibrated daily using both standard buffer and gases (BDH: $\mathrm{pH} 6.840 \pm 0.005$ at $37^{\circ} \mathrm{C}$ and $\mathrm{pH} 7.380 \pm 0.005$ at $37^{\circ} \mathrm{C}$ ) and tonometered blood (IL 237 tonometer). ${ }^{2}$ The machine is accurate within about $3 \%{ }^{3}$

In a further study we compared measurements obtained with each machine with those obtained from 40 duplicate arterial and capillary samples with the Corning 165.

Statistical methods-To compare values obtained with the individual machines and the Corning 165 and from arterial and capillary samples we used the overall test based on the null hypothesis described by Silvey. ${ }^{4}$ Mean values were compared by the paired $t$ test and standard deviations by the variance-ratio $(F)$ test. 